\title{
Aging impairs the hepatic subcellular distribution of ChREBP in response to fasting/feeding in rats: Implications on hepatic steatosis
}

\author{
Aurora Salamanca ${ }^{\mathrm{a}}$, Brenda Bárcena ${ }^{\mathrm{a}}$, Carmen Arribas a , Teresa Fernández-Agulló ${ }^{\mathrm{b}}$, Carmen Martínez ${ }^{\mathrm{b}}$, \\ José Ma. Carrascosa ${ }^{c}$, Manuel Ros ${ }^{b}$, Antonio Andrés ${ }^{\mathrm{a}, *, 1}$, Nilda Gallardo ${ }^{\mathrm{a}, *, 1}$

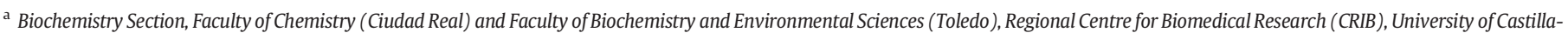 \\ La Mancha, Ciudad Real/Toledo, Spain \\ ${ }^{\mathrm{b}}$ Health Sciences Faculty, University Rey Juan Carlos, Alcorcón, Madrid, Spain \\ c Centre of Molecular Biology “Severo Ochoa", Autonomous University, Madrid, Spain
}

\section{A R T I C L E I N F O}

\section{Article history:}

Received 26 March 2015

Received in revised form 8 May 2015

Accepted 19 May 2015

Available online 21 May 2015

Section Editor: Holly M Brown-Borg

\section{Keywords:}

Aging

Hepatic steatosis

Insulin resistance

ChREBP

Nutritional adaptation

\begin{abstract}
A B S T R A C T
Aging is associated with alterations of lipid metabolism and increased prevalence of non alcoholic hepatic steatosis. Nevertheless, the mechanisms by which fat is accumulated in the liver during aging remain incompletely understood. In the present study, we investigated potential alterations that might contribute to the development of hepatic steatosis with aging. To this end, we analyzed the expression and the subcellular localization of key transcriptional factors involved in lipid metabolism such as ChREBP, Foxo1, Foxa2 and SREBP-1c in the liver of 3- and 24-month old Wistar rats. In addition, we studied the intracellular redistribution of ChREBP in response to fasting/refeeding transition. Old rats were characterized by hepatic steatosis, low serum ketone body levels and postprandial hyperinsulinemia. These observations were paralleled by the cytoplasmic localization and decreased expression of Foxa2, while ChREBP expression was markedly up-regulated and mainly localized in the nucleus. Consequently, the expression of lipogenic and $\beta$-oxidation genes was up-regulated or downregulated, respectively. Besides, the intracellular redistribution of ChREBP in response to fasting/refeeding transition was also impaired in old animals. Additionally, a negative correlation between serum ketone body levels and the nuclear localization of ChREBP was observed only in adult but not in old rats. Taken together, these data suggest that an age-related dysfunctional adaptation of ChREBP, in response to changes in the nutritional state, might contribute to the development of liver steatosis with aging.
\end{abstract}

(c) 2015 Elsevier Inc. All rights reserved.

\section{Introduction}

Aging is associated with an increase of adiposity, central and peripheral insulin resistance and a set of metabolic disorders with a common component: alteration of the caloric homeostasis and particularly the lipid metabolism. Consequently, hypertriglyceridemia and non alcoholic hepatic steatosis are frequent in the aged population (Gan et al., 2011). Despite extensive studies, the mechanisms responsible for excessive lipid accumulation in liver are incompletely understood (Trauner et al., 2010). However, obesity, insulin resistance and more recently the failure of leptin action have been

\footnotetext{
is Disclosure statement: All authors have nothing to declare.

* Corresponding authors at: Biochemistry Section, Faculty of Chemistry, CRIB, University of Castilla-La Mancha, 13071 Ciudad Real, Spain.

E-mail addresses: antonio.andres@uclm.es (A. Andrés), nilda.gallardo@uclm.es (N. Gallardo).

1 These authors contributed equally to this study.
}

considered among the primary metabolic disorders for the development of hepatic steatosis (Eriksson et al., 1986; Marchesini et al., 1999; Fishman et al., 2007).

Hepatic steatosis may arise from an imbalance between lipid deposition (from dietary fatty acid/glucose, peripheral lipolysis, and de novo lipogenesis) and elimination (via fatty acid oxidation and VLDLtriacylglyceride secretion) (Anderson and Borlak, 2008). These metabolic pathways are under tight regulation by the nutritionally regulated transcription factors ChREBP, SREBP-1c, Foxo1 and Foxa2, among others transcription factors. ChREBP and SREBP-1c are key lipogenic transcription factors responsible for the induction of gene encoding enzymes involved in fatty acid and triacylglyceride synthesis in the liver (Horton et al., 2002; Dentin et al., 2004). Accordingly, increased levels of ChREBP or SREBP-1c in the liver have been associated with hepatic steatosis in human and rodent models with extreme obesity, insulin resistance and type 2 diabetes (Shimomura et al., 1999; Dentin et al., 2006; Hurtado del Pozo et al., 2011). Furthermore, the subcellular distribution of ChREBP between the nucleus and cytosol is controlled by different metabolites including xylulose 5-phosphate, 
glucose 6-phosphate, fructose 2,6-biphosphate and ketone bodies (Kabashima et al., 2003; Arden et al., 2012; Dentin et al., 2012; Díaz-Moralli et al., 2012; Nakagawa et al., 2013).

The forkhead transcription factors, Foxo1 and Foxa2, belong to a superfamily of proteins that play a major role in regulating the metabolic functions of the liver (Le Lay and Kaestner, 2010). In fasting, Foxo1 increases the hepatic glucose production upregulating gene expression of two gluconeogenic enzymes, phosphoenolpyruvate carboxykinase and glucose-6-phosphatase catalytic subunit (Zhang et al., 2006). On the other hand, Foxa2 activates genes involved in fatty acid oxidation and ketone body production (Wolfrum et al, 2004). In the fed state, insulin stimulates the phosphorylation of Foxo1 and Foxa2 by Akt, resulting in nuclear exclusion and inhibition of their transcriptional activity (Biggs et al., 1999; Wolfrum et al., 2004). In this sense, in insulinresistant or hyperinsulinemic mice, Foxa2 is inactive and localized in the cytoplasm leading to lipid accumulation in the liver (Wolfrum et al., 2004), while in transgenic mice expressing constitutively active Foxo1 in the liver, de novo hepatic lipogenesis is decreased (Zhang et al., 2006). Despite the extensive knowledge on these transcription factors, the role of ChREBP, Foxa2, Foxo1 and SREBP-1c in the physiopathology of non alcoholic hepatic steatosis with aging remains to be fully elucidated.

Aged Wistar rats, as humans and other rodent models, present an increase of adiposity and develop insulin resistance (Escrivá et al., 1997, 2007). However, the insulin resistance of old rats is selective or tissue-specific, and does not uniformly develop and affect all target tissues and intracellular pathways at the same time. In fact, our previous studies demonstrated that the insulin signaling pathway that promotes glucose metabolism is impaired in visceral adipose tissue and skeletal muscle (Escrivá et al., 2007; de Solís et al., 2012), but not in the liver of old rats (Serrano et al., 2009). As a result, overnight fasted old Wistar rats are able to maintain normal serum glucose and insulin levels, but during oral glucose test or under euglycemic/hyperinsulinemic clamp conditions these animals need higher concentrations of insulin to cope with glucose, demonstrating its insulin resistance (Escrivá et al., 1997, 2007). Furthermore, several studies in humans and rodent models have shown that excessive lipid accumulation in the liver is not invariably associated with hepatic insulin resistance (Farese et al., 2012).

In this context, we have recently reported that old Wistar rats, besides hepatic steatosis, develop a mild perisinusoidal fibrosis associated with signs of oxidative stress and inflammation (Horrillo et al., 2013), but the involvement of critical transcription factors mediators of insulin and glucose signaling that sense fuel availability, such as ChREBP, Foxa2, Foxo1 and SREBP-1c, in age-associated hepatic steatosis was not addressed. In the present study, we hypothesized that alterations in these transcription factors underline the development of hepatic steatosis in old rats. To this end, we measured the expression levels and the subcellular distribution of these transcription factors, as well as, the expression of some target genes encoding enzymes of lipid synthesis and oxidation in liver with aging. We have also analyzed the adaptation of ChREBP in response to fasting and refeeding with aging, and the correlation among serum ketone body levels and the hepatic mRNA levels and nuclear protein localization of ChREBP in adult and old rats.

\section{Materials and methods}

\subsection{Animals}

Experiments were performed in male 3- and 24-month-old Wistar rats from our in-house colony (Centre of Molecular Biology, Madrid, Spain). Animals were housed in climate-controlled quarters with a 12-h light cycle and fed ad libitum standard laboratory chow and water. They were handled according to the European Union laws and the National Institute of Health $(\mathrm{NIH})$ guidelines for animal care.
The Institutional Advisory Committee of Bioethics and Animal Care reviewed and approved all the experimental procedures, and special care was taken to minimize animal suffering and to reduce the number of animals used.

\subsection{Analytical procedures}

All the animals were fasted $16 \mathrm{~h}$, including the overnight period, before being sacrificed by decapitation. Liver and visceral fat depots were carefully dissected and weighed. Then, tissues were flash frozen in liquid nitrogen and stored at $-70{ }^{\circ} \mathrm{C}$ until use. Serum samples were obtained by blood centrifugation and stored at $-70{ }^{\circ} \mathrm{C}$ until used. When examining the adaptation to the changes in the nutritional state, food was removed for $36 \mathrm{~h}$ prior to re-feeding animals for $3 \mathrm{~h}$, before euthanizing. In these animals serum samples were subsequently obtained in fasted or fed state.

Serum determinations were measured as previously described (Gallardo et al., 2007). Triacylglycerides (TAG) and total cholesterol levels were determined by enzymatic kits from Stanbio Laboratory (Boerne, TX). Non-sterified fatty acid (NEFA) levels were measured with an enzymatic assay from Wako Chemicals (Neuss, Germany) and glucose levels were determined using AmplexRed glucose/Glucose Oxidase Assay Kit (Molecular Probes, Inc., Eugene, OR). Total serum ketone body (KB) levels were quantified by an enzymatic kit from Wako Chemicals (Neuss, Germany). Serum insulin and leptin levels were measured by ELISA using rat specific kits from SPI-Bio (France), following the instructions of the manufacturer.

\subsection{Oral glucose tolerance test}

Oral glucose tolerance test (OGTT) was performed in overnight fasted rats as previously described (Escrivá et al., 2007). Briefly, 30\% glucose solution was administered intragastrically ( $2 \mathrm{~g} / \mathrm{kg}$ of body weight) and blood samples were obtained from the tail tip at 0,15, 30, 60 and 120 min after glucose administration. Glucose levels were measured immediately using an Accutrend Glucose Analyzer (Roche Diagnostics Corp., Indianapolis, IN). Serum insulin levels were assayed as described above. Overall changes in glucose and insulin during glucose tolerance test were calculated as the area under the curve (AUC) using the GraphPad prism version 6.01 for Windows (GraphPad Sotfware). The ratio of glucose AUC to insulin AUC was used as an index of whole body insulin sensitivity.

\subsection{Hepatic lipid measurements and staining techniques}

Total lipids were extracted from liver (100 mg) as previously described (Gallardo et al., 2007). The hepatic TAG and cholesterol content were analyzed with the same enzymatic kits used in serum analysis. For histological studies, liver specimens were taken immediately after the rats were sacrificed, incubated in fixation solution containing $4 \%$ paraformaldehyde and cryoprotected in $30 \%$ sucrose. $6 \mu \mathrm{m}$ serial frozen sections were taken in a cryomicrotome (Microm) and mounted onto gelatin-coated slides. Lipid staining was performed using the Oil Red O soluble dye (Sigma) that stains neutral lipids with an orange-red tint. Liver sections were stained as described (Goodpaster et al., 2000).

\subsection{Preparation of total, nuclear and cytoplasmic extracts from liver and immunoblot analysis}

$100 \mathrm{mg}$ of frozen liver was ground in the frozen state under liquid $\mathrm{N}_{2}$ before homogenization ( $2 \mathrm{ml}$ buffer/g tissue) in Henseleit buffer with $1 \mathrm{mM}$ PMSF, $2 \mathrm{mM} \mathrm{Na} \mathrm{VO}_{4}, 10 \mu \mathrm{g} / \mathrm{mL}$ leupeptin, $10 \mu \mathrm{g} / \mathrm{mL}$ aprotinin and $1 \mu \mathrm{g} / \mathrm{mL}$ pepstatin, using a manual Dounce homogenizer, and centrifuged at $800 \times g$ for $5 \mathrm{~min}$ at $4{ }^{\circ} \mathrm{C}$ to produce a total extract. NE-PER nuclear and cytoplasmic extraction reagents 
(Pierce Biotechnology, Rockford, IL) were used for preparing nuclear and cytoplasmic extracts from liver of 3- and 24-month old Wistar rats.

$100 \mu \mathrm{g}$ of protein from total and cytoplasmic extracts and $50 \mu \mathrm{g}$ of protein from nuclear extract were separated under reducing conditions in 7.5\% SDS-PAGE. Proteins were transferred to nitrocellulose sheets $(0.2 \mu \mathrm{m})$ (Bio-Rad, Spain) and incubated overnight at $4{ }^{\circ} \mathrm{C}$ with the corresponding primary antibody followed by incubation at room temperature for 30 min with secondary antibody conjugated with horseradish peroxidase. Primary rabbit polyclonal antibodies were anti-SREBP-1c (1:200; sc-8984, Santa Cruz Biotech., CA), anti-ChREBP (1:200; ab-30681, Abcam, Cambridge, UK), anti-ChREBP-ChIP grade (1:200; ab92809, Abcam, Cambridge, UK), anti-Foxo1a (1:500; ab39670, Abcam, Cambridge, UK), anti-p-S319-Foxo1a (1:1000; ab47326, Abcam, Cambridge, UK), anti-Foxa2 (1:500; ab-23630, Abcam, Cambridge, UK), anti-GW182 (1:500; ab-87541, Abcam, Cambridge, UK) and secondary antibody goat anti-rabbit conjugated with horseradish peroxidase (1:5000; ref-172-1019, Bio-Rad, Spain). Primary mouse monoclonal antibodies were anti-lamin A/C (1:1000; MAB-3538, Chemicon International, Millipore Corporation), anti- $\beta$-actin (1:5000; ab-8226, Abcam, Cambridge, UK) and secondary antibody goat antimouse conjugated with horseradish peroxidase (1:5000; ref-1706516, Bio-Rad, Spain).

The immunocomplexes formed were visualized using the ECL Western-blotting detection kit (Amersham Biosciences, Inc., Piscataway, NJ) with a G-Box Densitometer, and bands were quantified by scanning densitometry with exposure in the linear range using Gene Tools software (Syngene, Cambridge, UK), and normalized to that of $\beta$ actin. The densitometric relative levels of pS319-Foxo1 were normalized to the corresponding amount of Foxo1 protein mass in the same sample. Liver samples (total extracts, nuclear or cytosolic extracts) from 3- and 24-month old rats were run on the same gel to allow a direct comparison between the two age-groups. $\beta$-actin was used as control for protein loading. GW182 and lamin A/C were used as cytoplasmic and nuclear markers, respectively.

\subsection{RNA extraction and real-time RT-PCR}

Total RNA was isolated from liver as described (Gallardo et al., 2007). Relative quantitation of ChREBP, Foxo1a, Foxa2, SREBP-1c, acetyl-CoAcarboxylase- $\alpha$ (ACC $\alpha$ ), fatty acid synthase (FAS), diacylglycerol acyltransferase-1 (DGAT-1), diacylglycerol acyltransferase-2 (DGAT2 ), microsomal transfer protein (MTTP), peroxysome proliferator activating receptor- $\alpha$ (PPAR- $\alpha)$, PPAR $\gamma$-coactivator- $1 \alpha$ (PGC- $1 \alpha)$ and carnitine palmitoyl transferase 1a (CPT-1a) mRNA expression was performed by TaqMan real-time PCR according to the manufacturer's protocol on an ABI PRISM 7500 FAST Sequence Detection System instrument and software (PE Applied Biosystems, Foster City, CA), using PreDeveloped TaqMan Assay Reagents (PE Applied Biosystems) (Supplementary Table 1 ). To normalize the amount of sample cDNA added to the reaction, amplification of endogenous control 18S rRNA was included in separate wells as real-time reporter. The $\Delta \Delta C_{T}$ method was used to calculate the relative differences between experimental conditions and control groups as fold change in gene expression (Livak and Schmittgen, 2001).

\subsection{Statistics}

Data are expressed as mean \pm SEM. The significance of differences between two groups was determined by unpaired Student's t-test. Correlation analysis was determined by Pearson's correlation coefficient test (r). Statistical analysis was performed using the GraphPad Prism version 6.01 for Windows (GrapPad Software). Differences were considered significant at $p<0.05$.
Table 1

Body weight and serum metabolic parameters in overnight fasted 3- and 24-month-old rats.

\begin{tabular}{lcc}
\hline & 3-month & 24-month \\
\hline Body weight $(\mathrm{g})$ & $363 \pm 7$ & $672 \pm 17^{*}$ \\
Visceral fat $(\mathrm{g})$ & $9.9 \pm 2.7$ & $26.7 \pm 2.8^{*}$ \\
Serum glucose $(\mathrm{mmol} / \mathrm{L})$ & $4.5 \pm 0.2$ & $5.0 \pm 0.1$ \\
Serum insulin $(\mathrm{ng} / \mathrm{ml})$ & $1.32 \pm 0.2$ & $1.36 \pm 0.2$ \\
Serum leptin $(\mathrm{ng} / \mathrm{ml})$ & $4.8 \pm 0.8$ & $16.5 \pm 2.6^{*}$ \\
Serum TAG $(\mathrm{mg} / \mathrm{dl})$ & $90 \pm 4$ & $171 \pm 8^{* *}$ \\
Serum cholesterol $(\mathrm{mg} / \mathrm{dl})$ & $59 \pm 2$ & $138 \pm 8^{* *}$ \\
Serum NEFA (mmol/L) & $1.01 \pm 0.04$ & $1.08 \pm 0.03$ \\
Serum ketone bodies $(\mathrm{mmol} / \mathrm{L})$ & $0.72 \pm 0.09$ & $0.31 \pm 0.01^{*}$ \\
\hline
\end{tabular}

Data were obtained from 8 to 10 rats per group and represent mean \pm SEM. Biochemical determinations were made in duplicate $\left({ }^{*} p<0.05\right.$; ${ }^{* *} p<0.005$ compared to 3-month-old rats). TAG: triacylglycerides; NEFA: non-sterified fatty acid.

\section{Results}

\subsection{Biological characteristics of 3- and 24-month old rats}

Body weight and metabolic parameters of the animals used in the present experiments are shown in Table 1. Old Wistar rats exhibit increased body weight, visceral fat mass and serum leptin levels. Overnight fasted old rats had normal serum glucose and insulin levels, and no significant effect of age was also observed in NEFA levels. Nevertheless, old animals became hypertriglyceridemic and hypercholesterolemic, but according to our earlier observations (Escrivá et al., 1997), the levels of ketone bodies were significantly decreased compared with adult mature rats (Table 1). Changes in glucose and insulin levels during OGTT are shown in Fig. 1. The increment of glucose was similar for the two age groups indicating that glucose tolerance was not modified during aging. In contrast, the curve for insulin in response to the glucose load increased significantly in the old rats, suggesting the development of peripheral insulin resistance. Consistent with this, the insulin sensitivity index, calculated as the ratio of glucose AUC to insulin AUC, decreased significantly in old rats confirming our previous observations (Escrivá et al., 2007).

\subsection{Increased lipid content in liver from old rats}

As shown in Fig. 2A, old Wistar rats accumulated significantly higher levels of TAG and cholesterol in the liver. The excessive accumulation of neutral lipids in the livers from old rats was confirmed upon Oil Red 0 staining, while lipid droplet staining was absent in the young animals (Fig. 2B). Moreover, the liver-to-body weight ratio (\%) was increased in old compared with adult rats $(3.27 \pm 0.13 v s$. $2.81 \pm 0.06$, respectively; $\mathrm{n}=10, \mathrm{p}<0.05$ ).

\subsection{Up-regulation of hepatic ChREBP expression in old rats}

To determine whether aging alters the expression levels of ChREBP, Foxa2, Foxo1 and SREBP-1c in the liver of rats, we measured the mRNA and total protein levels of these transcription factors. As can be seen in Fig. 3A, we found a $\sim 2.0$ fold increase in the relative mRNA levels of ChREBP in old rats, while there was no change in the mRNA levels of SREBP-1c between the two age-groups. On the other hand, we observed that the mRNA levels of the FKH factors, Foxo1 and Foxa2, were also altered in the liver from old rats. While the transcript levels of Foxo1 were slightly increased $\sim 1.6$ folds, Foxa 2 mRNA levels were decreased $\sim 2.0$ folds in old compared with adult mature rats (Fig. 3A). Immunoblot analysis was used to determine the protein abundance of these transcription factors in total liver extracts from 3- and 24-month old rats. Fig. 3B shows that in parallel to the mRNA levels, ChREBP total protein levels were significantly increased, while Foxa2 protein levels were decreased in the liver from old rats. However, the total protein levels of 

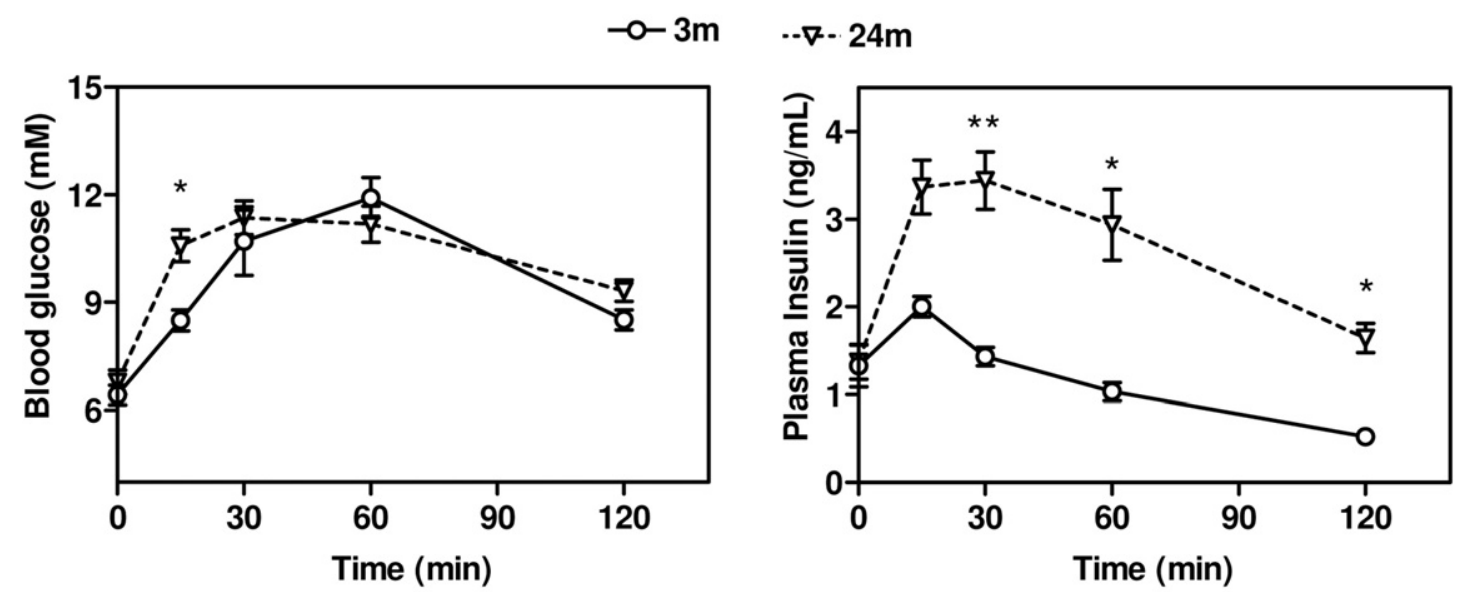

\begin{tabular}{|l|c|c|c|}
\hline & Glucose AUC & Insulin AUC & $\begin{array}{c}\text { Insulin sensitivity index } \\
\text { (Glucose AUC / Insulin AUC) }\end{array}$ \\
\hline $3 \mathrm{~m}$ & $429 \pm 50$ & $4.8 \pm 1.1$ & $91 \pm 13$ \\
\hline $24 \mathrm{~m}$ & $429 \pm 27$ & $27.6 \pm 4.0^{* *}$ & $18.6 \pm 3.0^{* *}$ \\
\hline
\end{tabular}

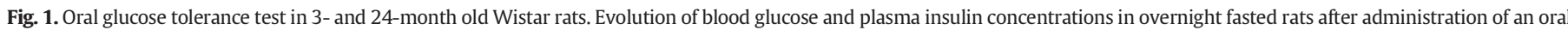

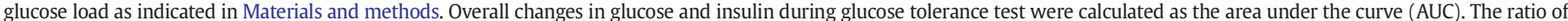

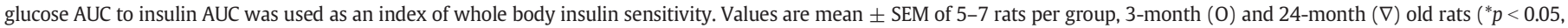
${ }^{* *} p<0.01$ vs. 3-month old rats).

hepatic SREBP-1c (125 kDa) and Foxo1 did not change with aging (Fig. 3B).

\subsection{Effect of aging in the expression of genes involved in hepatic lipid} metabolism

Next, we have analyzed the mRNA levels of several hepatic genes involved in lipid metabolism under the control of the transcription factors mentioned above. In agreement with the observed changes in hepatic ChREBP expression in aging rats, the mRNA levels of the lipogenic enzymes ACC- $\alpha$ and FAS were significantly up-regulated; 2.0 and 3.1 folds respectively, in old rats (Table 2). We have also observed that aging caused a significant increase in the mRNA abundance of both, DGAT1 and DGAT2, and MTTP (Table 2), suggesting an increased esterification and VLDL-TAG secretion in aged rats. Finally, we determined the expression levels of PPAR $\alpha$, PGC- $1 \alpha$ and CPT-1a involved in lipid
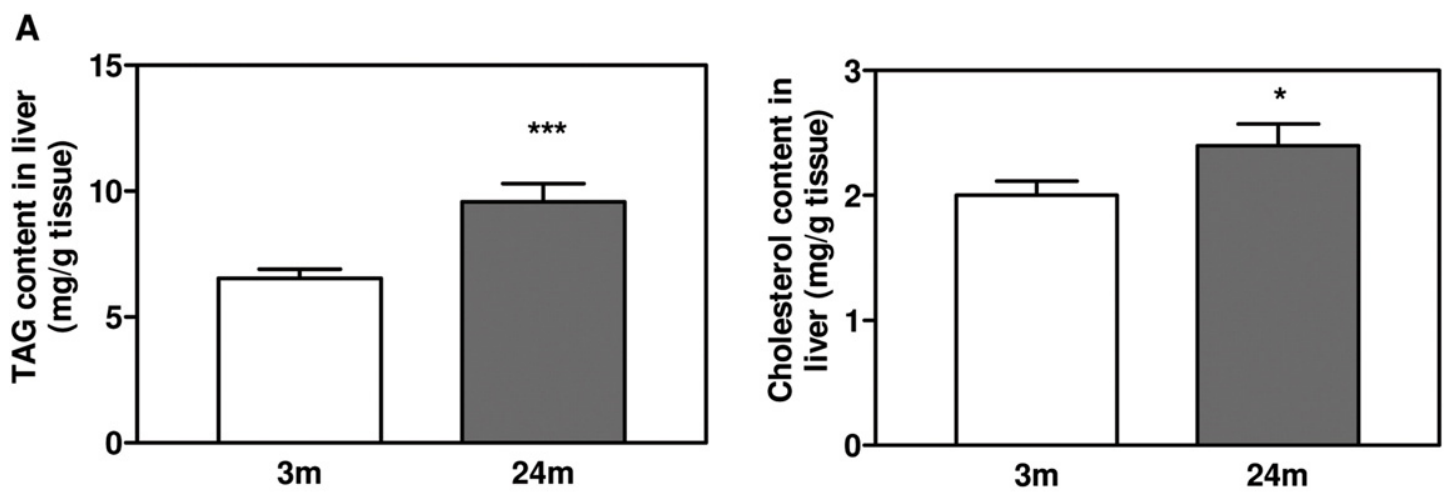

B

$3 m$

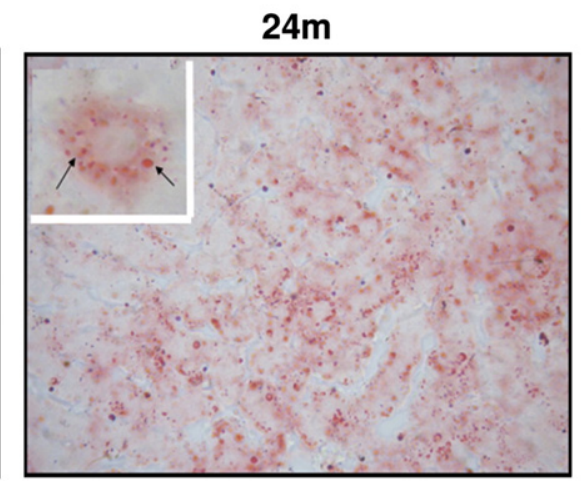

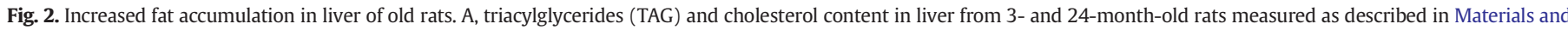

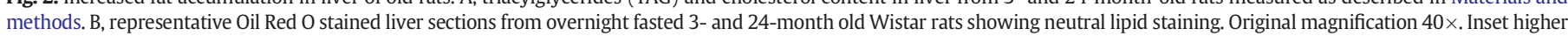
magnification shows lipid droplets $100 \times$. Results are the mean \pm SEM of 10 rats per group $\left({ }^{*} p<0.05,{ }^{* * *} p<0.001\right.$ compared to 3 -month-old rats). 
A

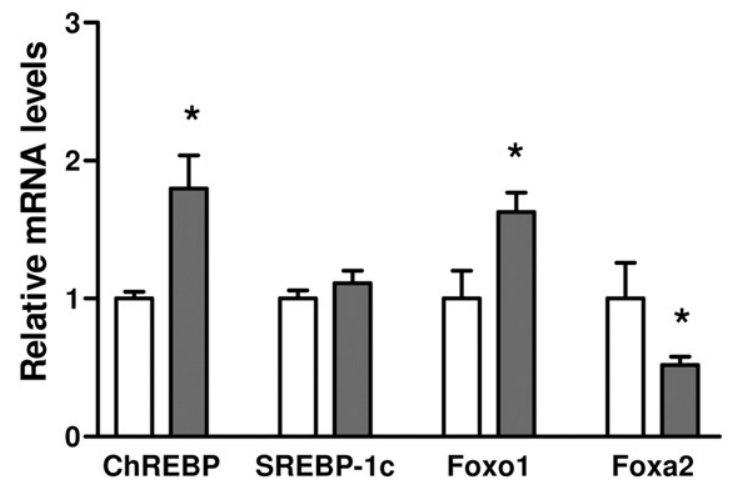

B Total extract
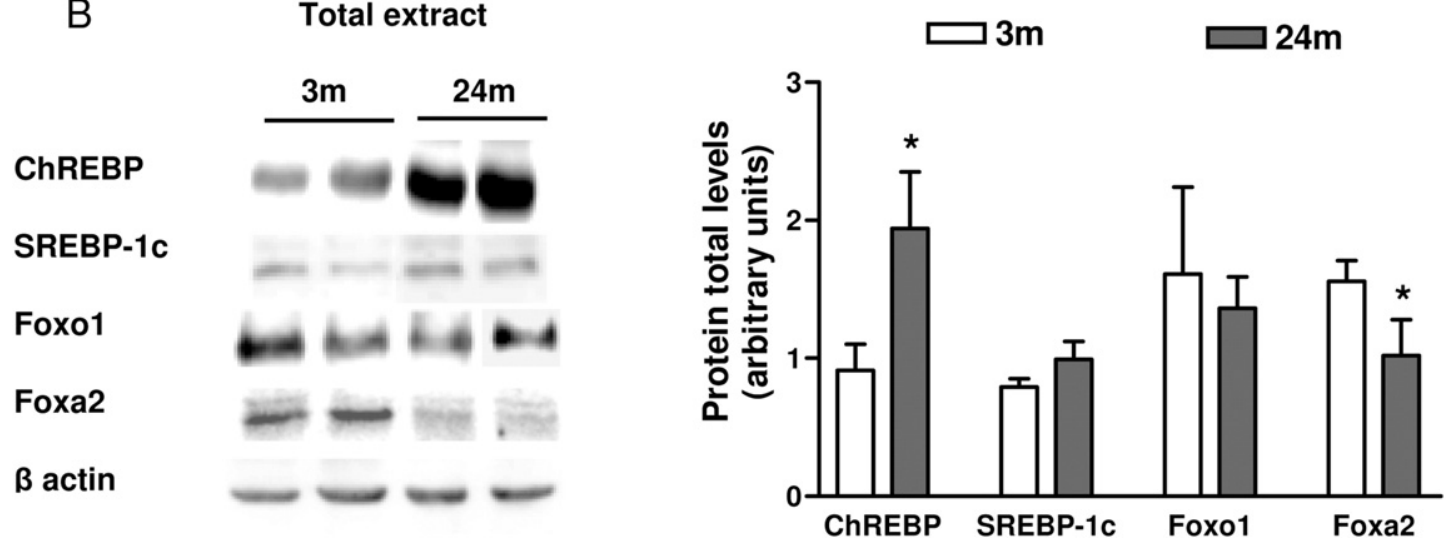

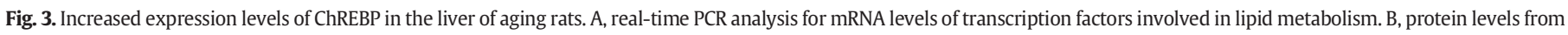

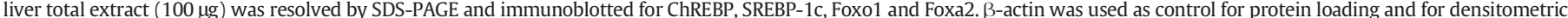

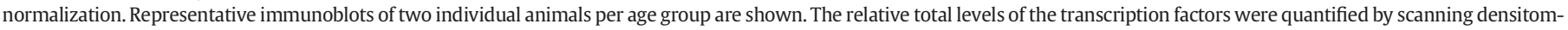
etry. Data are the means \pm SEM of 5 separate determinations per age group ( ${ }^{*} p<0.05 v s$. 3-month old rats).

oxidation. Whereas the mRNA levels of PPAR $\alpha$ and PGC- $1 \alpha$ increased $\sim 1.4$ and $\sim 2.6$ folds, respectively, the expression levels of CPT-1a, the most important enzyme for controlling the availability of long-chain acyl-CoA for mitochondrial fatty acid oxidation, was markedly decreased $\sim 2.5$ folds in old rats (Table 2 ).

3.5. Effect of aging in the hepatic subcellular distribution of ChREBP, Foxo1, Foxa2 and SREBP-1C

In order to study the effect of aging on the hepatic subcellular localization of these transcriptions factors, we analyzed their relative distribution between cytoplasm and nucleus isolated from the liver of overnight fasted 3- and 24-month old rats. Interestingly, we found

Table 2

Changes in gene expression of several genes involved in lipid metabolism in the liver in 3 and 24-month-old Wistar rats using $18 \mathrm{~S}$ rRNA as the invariant control.

\begin{tabular}{lll}
\hline Gene & 3-month & 24-month \\
\hline & Lipogenesis & \\
ACC- $\alpha$ & $1 \pm 0.1$ & $2.0 \pm 0.1^{* *}$ \\
FAS & $1 \pm 0.2$ & $3.1 \pm 0.3^{* *}$ \\
& Lipid esterification and secretion & \\
DGAT-1 & $1 \pm 0.01$ & $4.0 \pm 0.1^{* *}$ \\
DGAT-2 & $1 \pm 0.04$ & $3.1 \pm 0.6^{* *}$ \\
MTTP & $1 \pm 0.05$ & $1.9 \pm 0.2^{* *}$ \\
& Lipid oxidation & \\
PPAR $\alpha$ & $1 \pm 0.1$ & $1.4 \pm 0.1^{*}$ \\
PGC-1 $\alpha$ & $1 \pm 0.1$ & $2.6 \pm 0.1^{* *}$ \\
CPT-1a & $1 \pm 0.07$ & $0.4 \pm 0.05^{* *}$ \\
\hline
\end{tabular}

Data are the mean \pm SEM of 4 to 5 separate determinations, made in triplicate $\left({ }^{*} p<0.05\right.$; ${ }^{* *} p<0.005$ compared to 3 -month-old rats). that the nuclear protein levels of ChREBP in overnight fasted old rats were significantly increased $\sim 3$ folds as compared with adult rats (Fig. 4, A and C). Our data also show that, the cytosolic protein levels of ChREBP decreased $\sim 6$ folds in old compared to adult control rats (Fig. 4, B and C). These findings are in line with recent data suggesting that the nuclear localization of ChREBP can be inhibited in isolated hepatocytes incubated with ketone bodies (Nakagawa et al., 2013). Thus, as serum ketone body levels in old rats are significantly lower than in adult rats (Table 1), this would explain the high nuclear content of ChREBP found in the liver from old rats.

On the other hand, the nuclear protein levels of Foxo1 and Foxa2 were significantly decreased in overnight fasted old rats (Fig. 5A). Particularly, the content of Foxa2 in the nuclear fraction of liver extracts from old rats was barely visible (Fig. 5A) and decreased $\sim 4.5$ folds as compared to adult rats (Fig. 5D), and this was followed by a marked increase in the cytosol in old rats (Fig. 5, B and D). In addition, there is a slight decrease in the cytosolic protein content of Foxo1 (Fig. 5B), while the phosphorylated Foxo1 levels increased $\sim 2.1$ folds in old rats (Fig. 5, B and C). Finally, the nuclear protein levels of the SREBP-1c (68 kDa) remain unchanged with aging (Supplementary Fig. 1).

3.6. Dysfunctional adaptation of hepatic ChREBP in response to fasting and feeding in aging rats

Because the inhibition of nuclear localization of ChREBP is closely related with the production of ketone bodies (Nakagawa et al., 2013), we sought to analyze if increasing serum ketone body levels in old rats would decrease the nuclear localization of ChREBP. To this end, as serum ketone bodies are increased upon prolonged starvation, we studied the subcellular distribution of ChREBP in response to changes 
A

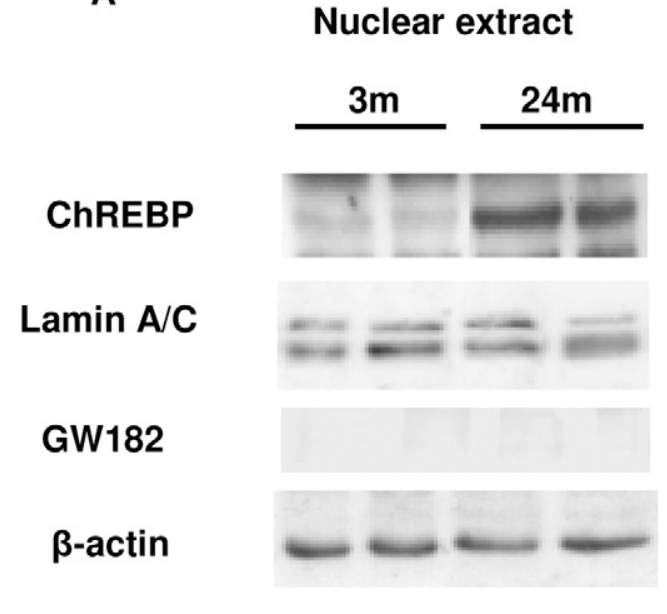

B

\section{Cytoplasmic extract}

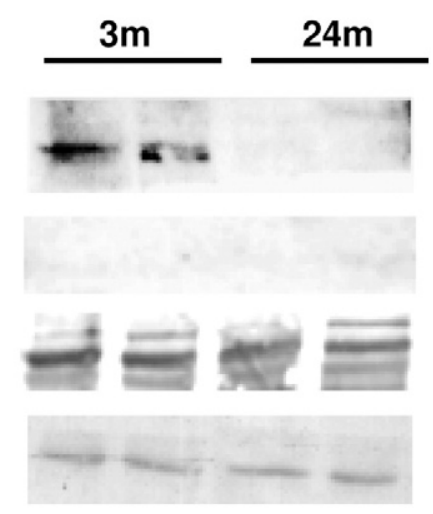

C Nuclear levels Cytoplasmic levels

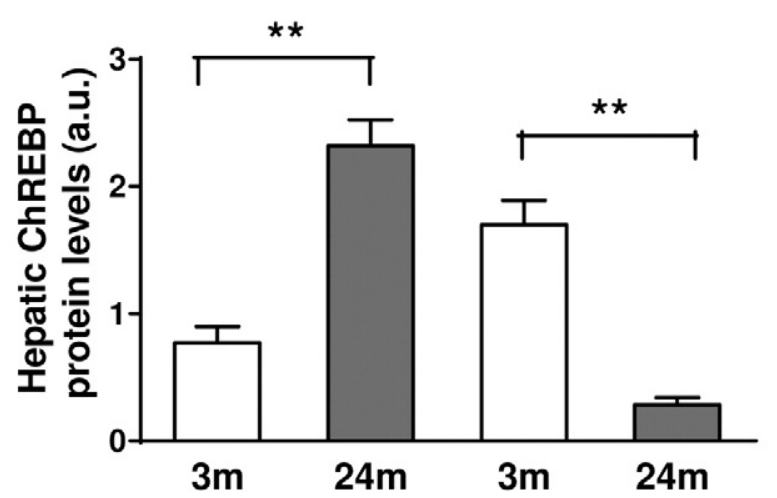

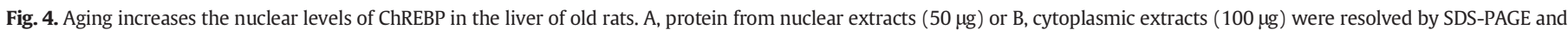

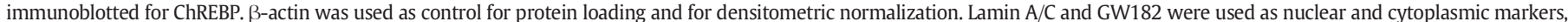

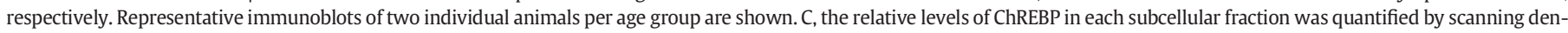
sitometry. Data are the means \pm SEM of 5 separate determinations per age group ( ${ }^{* *} p<0.005 v$ s. 3-month old rats).

in the nutritional state, in rats fasted for $36 \mathrm{~h}$ and then re-fed during $3 \mathrm{~h}$ before killing the animals. As expected, upon a prolonged fasting, there was a marked increase in serum ketone body levels in 3- and 24-monthold rats ( $1.94 \pm 0.2$ vs. $1.51 \pm 0.17 \mathrm{mmol} / \mathrm{L}$, respectively; $\mathrm{n}=4-6$; n.s. $)$ (Table 3), compared to the levels of overnight fasted adult and old animals $(0.72 \pm 0.09$ vs. $0.31 \pm 0.01 \mathrm{mmol} / \mathrm{L}$, respectively; $\mathrm{n}=(8-10$; $\left.{ }^{*} p<0.05\right)$ ) (Table 1). Accordingly, we found decreased mRNA (Fig. 6A) and nuclear protein levels of ChREBP (Fig. 6B) in liver from adult rats. In contrast, despite the high increase in serum ketone bodies levels in old rats reaching similar concentration than those in adult rats upon $36 \mathrm{~h}$ starvation, the mRNA (Fig. 6A) and nuclear protein localization of ChREBP (Fig. 6B) remain high in the liver of aging rats. Taken together, these data might suggest that the inhibition of hepatic nuclear localization of ChREBP by ketone bodies is impaired in old rats.

On the other hand, in response to re-feeding, there was a significant increase in the mRNA and nuclear protein levels of ChREBP (Fig. 6, A and B) in adult rats, consistent with the marked decrease in ketone body levels $\sim 13.8$ folds (Table 3 ) in these animals. In contrast, the transition from fasting to re-feeding in old rats induced a small, not significant, increase over the already high mRNA and nuclear protein levels of ChREBP (Fig. 6, A and B) that parallels the low decrease $\sim 2.2$ folds in serum ketone body levels found in aging rats (Table 3 ). In addition, to analyze the association between serum ketone body levels and hepatic ChREBP, we performed correlation analysis. As illustrated, a significant negative correlation between serum ketone body levels and hepatic mRNA levels $(\mathrm{r}=-0.903, \mathrm{p}<0.003 ;$ Fig. $6 \mathrm{~A})$ and hepatic nuclear protein levels of
ChREBP $(\mathrm{r}=-0.827, \mathrm{p}<0.003$; Fig. $6 \mathrm{C}$ ) was only observed in adult rats, whereas no correlation was found in old rats (Fig. 6, A and C). Finally, it is worthy to note that the increment on serum glucose, upon re-feeding, was similar for the two age groups of rats, but serum insulin concentrations were significantly higher in old compared with adult rats (Table 3). These data sustain that prolonged fasting exacerbates the insulin resistance in old rats and that higher insulin levels were required to maintain normal levels of fasting serum glucose in aging rats.

\section{Discussion}

Aging is a physiological condition characterized by certain metabolic disorders associated with obesity, insulin resistance, and dyslipidemia that give rise to hepatic steatosis (Trauner et al., 2010; Gan et al., 2011). Among the mechanisms that lead to hepatic steatosis are increased lipogenesis and reduced $\beta$-oxidation (Diraison et al., 2003; Tamura and Shimomura, 2005). Thus, our aim in the present study was to investigate whether alterations in the expression and/or subcellular distribution of ChREBP, Foxa2, Foxo1 and SREBP-1c, key transcriptions factors involved in liver lipid metabolism, underline the development of steatosis with aging, a physiological model characterized by mild increased adiposity and insulin resistance as compared with models with extreme obesity, insulin resistance and type 2 diabetes as the $o b / o b$ mice and/or diet-induced obese mice and rats 

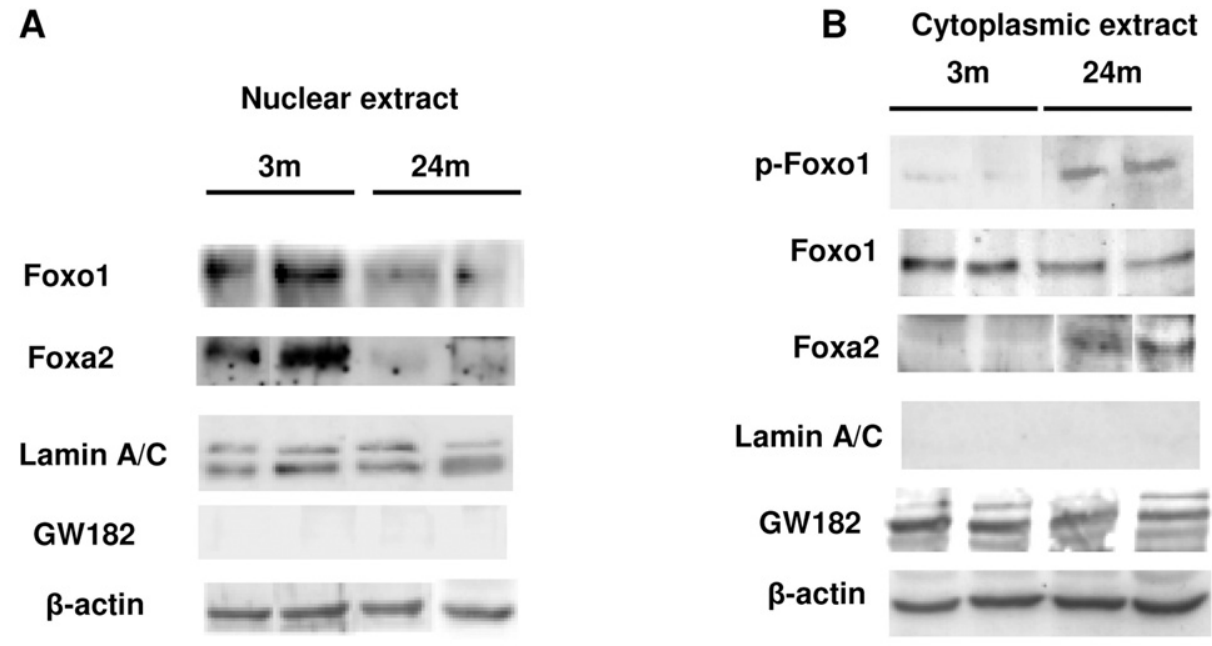

C

Hepatic Foxo1 subcellular distribution

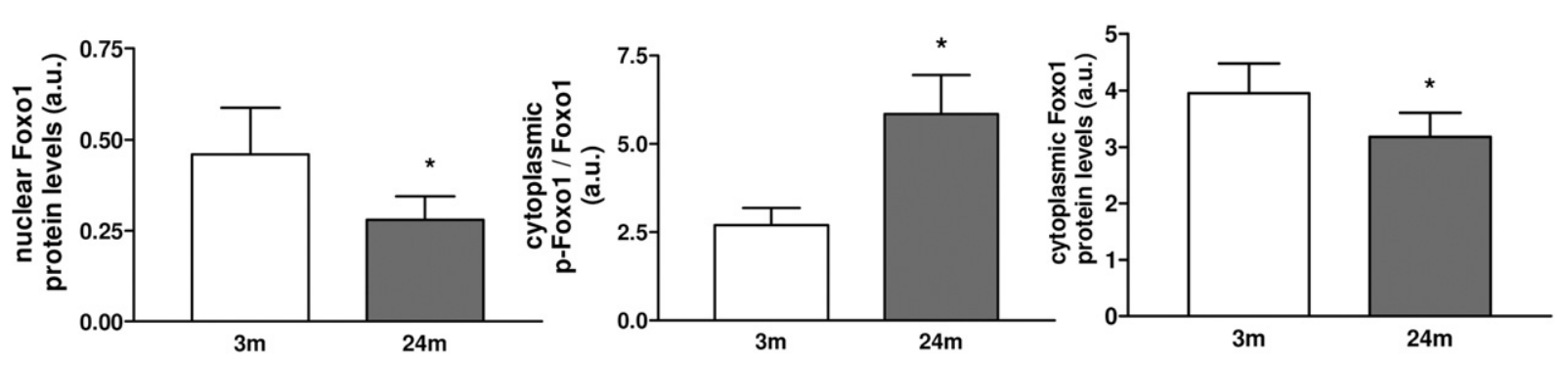

D

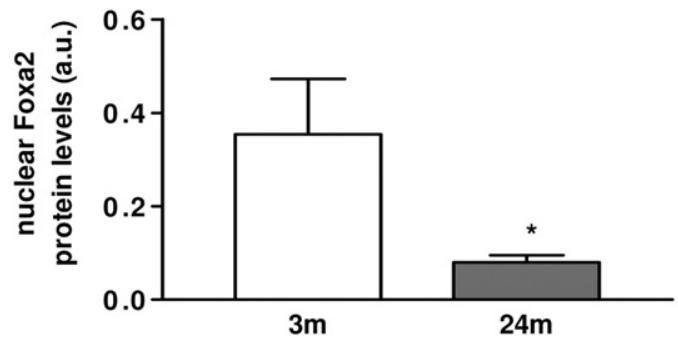

Hepatic Foxa2 subcellular distribution

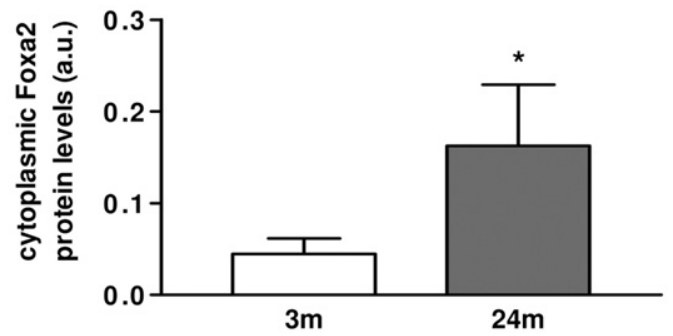

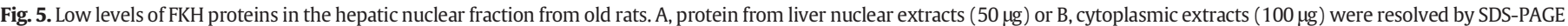

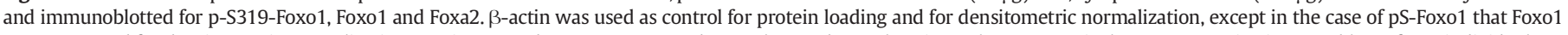

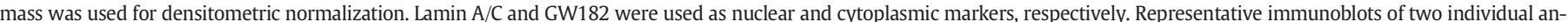

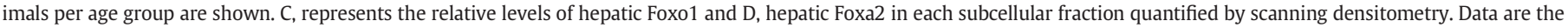
means \pm SEM of 5 separate determinations per age group $\left({ }^{*} p<0.05\right.$ vs. 3 -month old rats).

(Shimomura et al., 1999; Iizuka et al., 2004; Wolfrum et al., 2004; Dentin et al., 2006).

We show that hepatic TAG and cholesterol content were markedly increased in liver from old animals. Besides, we also show that old rats, in order to maintain normoglycemia, need higher concentrations of insulin compared with adult rats upon an OGTT, a situation that was also observed when animals were fed after prolonged fasting. These data demonstrate the presence of postprandial hyperinsulinemia as a result of the insulin resistance with aging. As the insulin resistance appears in visceral adipose tissue and skeletal muscle (Escrivá et al., 2007; de Solís et al., 2012), but not in liver of old rats (Serrano et al., 2009), this selective or tissue-specific peripheral insulin resistance

Table 3

Serum metabolic parameters in 3- and 24-month-old rats in response to fasting and feeding.

\begin{tabular}{|c|c|c|c|c|}
\hline & \multicolumn{2}{|l|}{ 3-month } & \multicolumn{2}{|l|}{ 24-month } \\
\hline & 36 h fast & $3 \mathrm{~h}$ fed & 36 h fast & $3 \mathrm{~h} \mathrm{fed}$ \\
\hline Serum glucose (mmol/L) & $4.02 \pm 0.1$ & $6.33 \pm 0.34^{++}$ & $5.24 \pm 0.8$ & $6.74 \pm 1.32^{++}$ \\
\hline Serum insulin $(\mathrm{ng} / \mathrm{ml})$ & $0.42 \pm 0.05$ & $2.48 \pm 0.31^{++}$ & $2.15 \pm 0.32^{* * *}$ & $4.15 \pm 0.19^{* *},++$ \\
\hline Serum NEFA (mmol/L) & $0.68 \pm 0.1$ & $0.43 \pm 0.05^{++}$ & $0.71 \pm 0.06$ & $0.56 \pm 0.09$ \\
\hline Serum total KB (mmol/L) & $1.94 \pm 0.2$ & $0.14 \pm 0.02^{+++}$ & $1.51 \pm 0.17$ & $0.68 \pm 0.04^{* * *},+$ \\
\hline
\end{tabular}

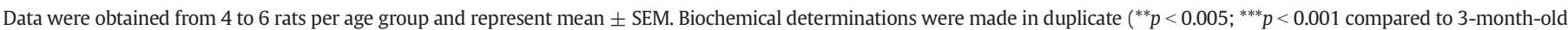
rats); $\left({ }^{+} p<0.05 ;{ }^{++} p<0.005 ;{ }^{++} p<0.001\right.$ compared to same age fed). NEFA: non-sterified fatty acid; KB: ketone bodies. 

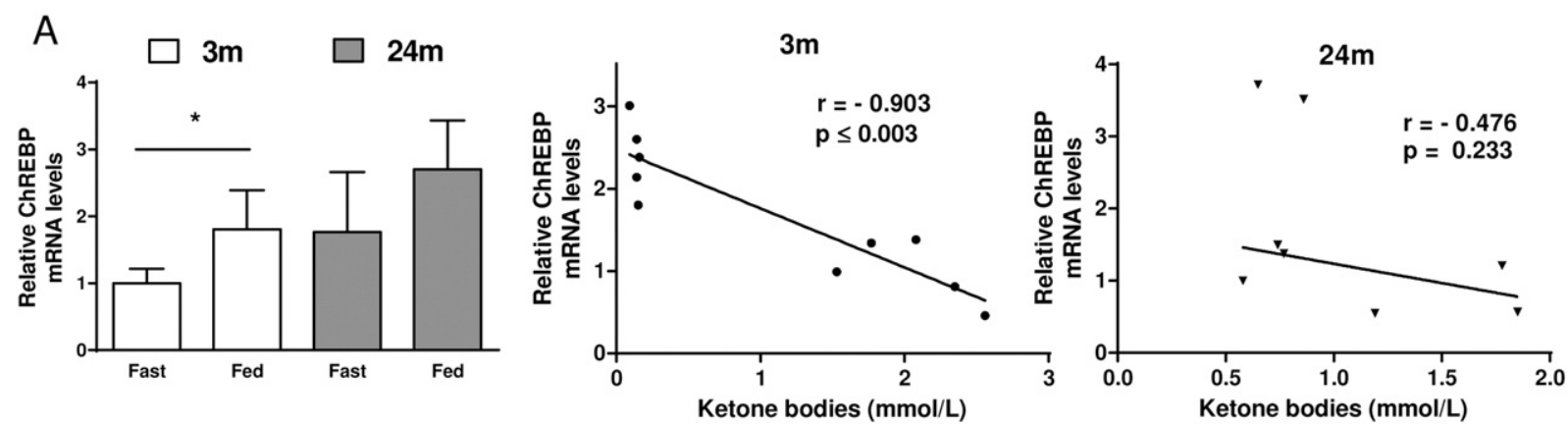

B

\section{Nuclear extract}

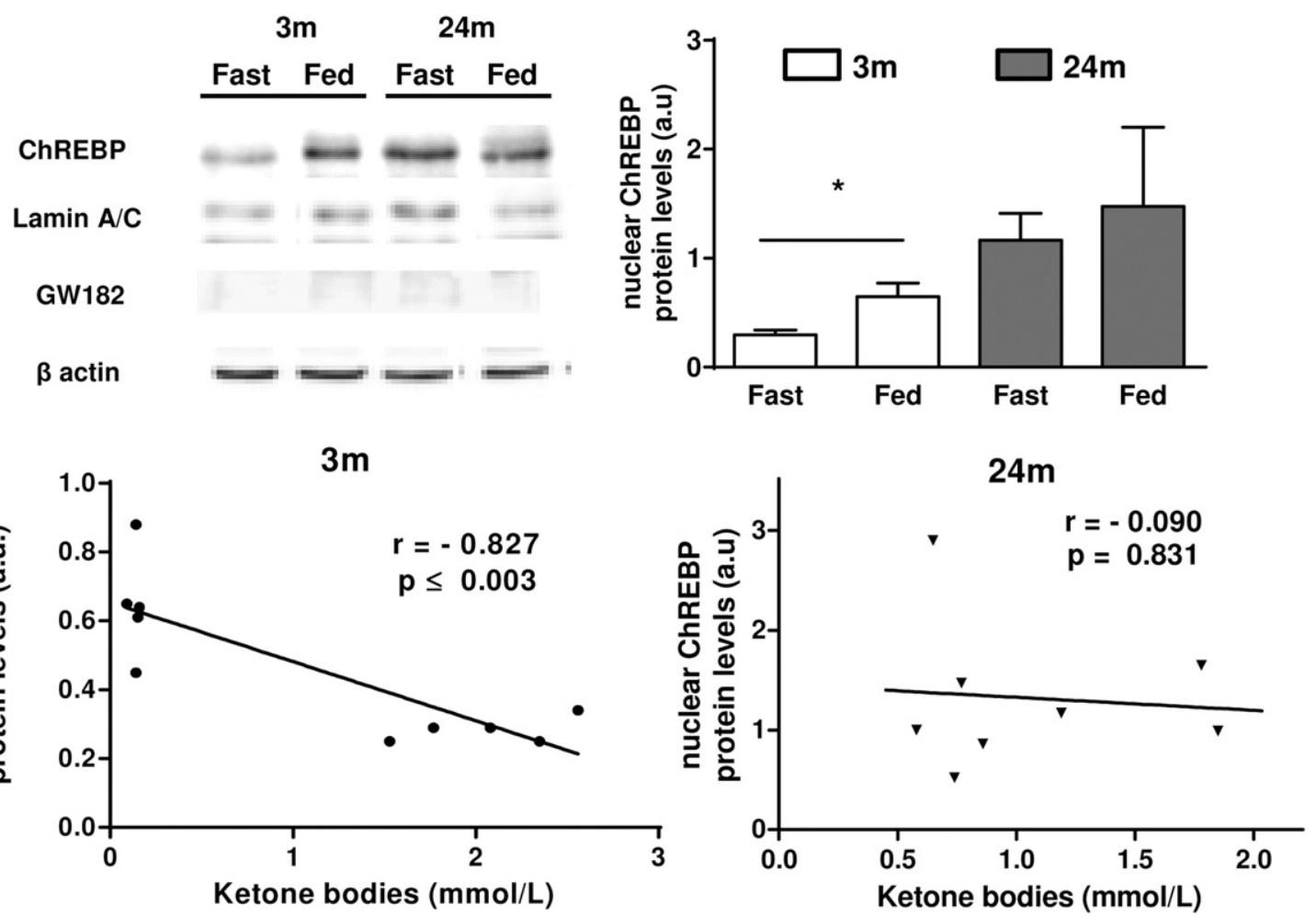

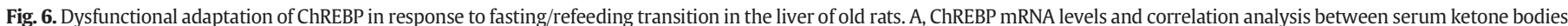

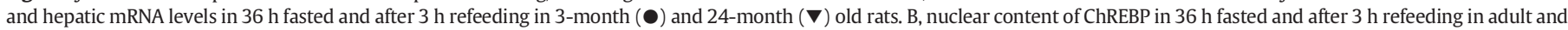

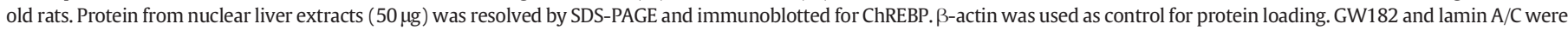

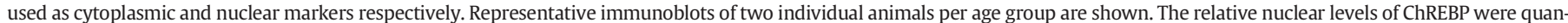

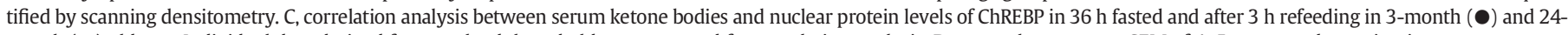

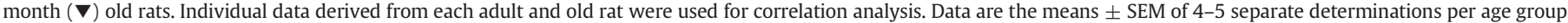
( ${ }^{*} p<0.05$ vs. 3-month old rats).

could well contribute to cause hepatic steatosis by increasing postprandial availability of FA and glucose for hepatic de novo lipogenesis in aging rats probably due to altered nutrient handling by the insulin resistant peripheral tissues (Otero et al., 2014). Indeed, it has been shown that skeletal muscle insulin resistance in human aging promotes increased hepatic steatosis by decreasing postprandial glucose storage away from muscle glycogen and increasing glucose delivery toward de novo lipogenesis in the liver (Flannery et al., 2012) and that the excessive lipid accumulation in the liver is not invariably associated with hepatic insulin resistance (Farese et al., 2012).

At molecular level, we found, for the first time, a significant decrease in the expression and nuclear content of Foxa2, while the cytoplasmic, inactive, Foxa2 protein levels were increased in the liver from insulinresistant old rats. These data are in line with previous observations in hyperinsulinemic insulin-resistant $o b / o b$ mice and diet-induced obese mice, characterized by the cytoplasmic localization and inactivation of
Foxa2 in the liver (Wolfrum et al., 2004). Therefore, the persistent postprandial hyperinsulinemia in old animals reported herein might stimulate the cytoplasmic translocation of Foxa2 impairing ketogenesis in the liver in aging rats. This suggestion is supported by several lines of evidence. First, it has been previously reported that prolonged exposure of primary hepatocytes to insulin decreased ketogenesis (Agius et al., 1986) and reduced the transcript levels of CPT-1a whereas increased hepatic lipogenic program (Liu et al., 2009). Secondly, a decreased ketogenesis in primary hepatocytes incubated with oleate in aging rats has been reported (Sastre et al., 1996). Finally, in agreement with these data we found a significant down-regulation of hepatic CPT-1a gene expression, increased expression of genes involved in lipogenesis such as ChREBP, ACC- $\alpha$ and FAS, and decreased serum ketone body levels in old compared with adult rats.

Akt-mediated phosphorylation of Foxo1 in the fed state results in its exclusion from the nucleus and inhibition of its transcriptional activity 


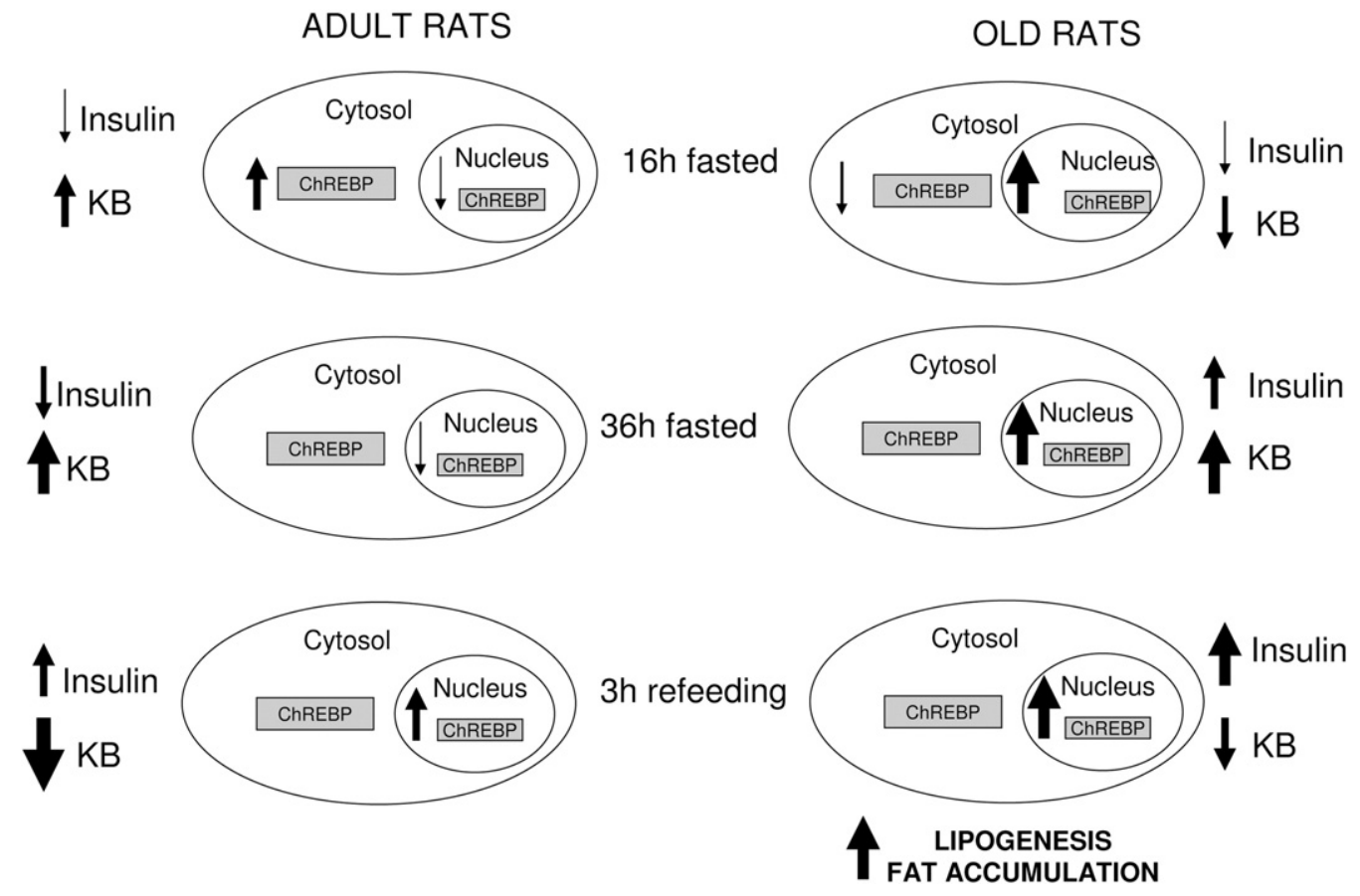

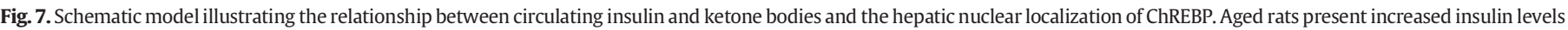

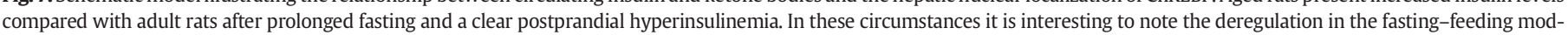

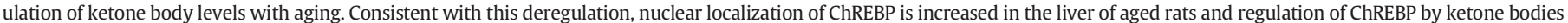
appears to be affected. As a result, the lipogenesis and fat accumulation in the liver of the aged rats are increased.

(Biggs et al., 1999). Nevertheless, we have previously reported increased basal Akt activity in livers from overnight fasted old rats (Serrano et al., 2009) that may well explain the high phosphorylation levels of Foxo1 in the cytoplasm, leading to the inhibition of Foxo1 transcriptional activity with aging. Thus, it might be suggested that the cytoplasmic localization of Foxo1 could be linked to the increased total and nuclear protein levels of ChREBP found in old rats. In fact, it has been reported that liver specific Foxo1 knockout mice enhanced total protein levels of ChREBP and recruitment to its target gene promoter (IdoKitamura et al., 2012).

SREBP-1c has been considered a key mediator for insulin resistance and hepatic steatosis (Moon et al., 2012) and overproduction of nuclear-active SREBP-1c inhibited IRS2/PI3K/AKT pathway, decreased glycogen synthesis and promoted lipogenesis in the liver (Ide et al., 2004). In this work, the expression of SREBP-1c and the levels of its mature form in the nucleus of the overnight fasted rats were not altered with aging. Although SREBP-1c is responsible for the induction of lipogenic genes ACC- $\alpha$ and FAS, which expression is also increased synergistically by ChREBP (Dentin et al., 2004), this transcription factor does not seem to be involved in the development of the hepatic steatosis with aging. In line with this suggestion, it has been shown that ChREBP, but not SREBP-1c, was increased in the liver nuclear fraction in $24 \mathrm{~h}$ fasted $o b / o b$ mice (Dentin et al., 2006). Nevertheless, the role of SREBP-1c in hepatic steatosis with aging needs further work before a final conclusion may be drawn.

It is known that ChREBP ${ }^{-1-}$ mice resulted in decreased expression of key lipogenic enzymes, leading to a significant decrease in hepatic fatty acid synthesis rates compared to wild-type mice (Iizuka et al., 2004). Likewise, an important reduction in hepatic lipogenesis was also shown in double deficient ob/ob-ChREBP ${ }^{-1-}$ mice (Iizuka et al., 2006). Collectively, these data suggested a potential role of ChREBP in the development of hepatic steatosis. In fact, recent data show that ChREBP overexpressing mice fed a high-fat diet are characterized by increased expression of genes involved in lipogenesis, fatty acid esterification and TAG mobilization, such as ACC- $\alpha$, FAS, DGAT- 1 and MTTP, leading to hepatic steatosis (Benhamed et al., 2012). In this study, we show that the increased levels and nuclear localization of ChREBP in livers from old rats was paralleled by the significant up-regulation of their lipogenic target genes ACC- $\alpha$ and FAS, as well as DGAT-1, DGAT- 2 and MTTP. According to this data and because the hepatic MTTP expression is enhanced by Foxo1 and Foxa2 (Wolfrum and Stoffel, 2006; Kamagate et al., 2008), it is tempting to speculate that the up-regulation and nuclear localization of ChREBP, and its probably increased transcriptional activity, would increase MTTP expression reported herein in old rats, compensating in this way for the inactivation of Foxo1 and Foxa2 that should lead to the downregulation of MTTP expression. In this regard, it has been shown that the heterodimer formed by ChREBP and Maxlike protein X (ChREBP-MlX), the principal transcription factor regulating glucose-responsive lipogenic enzyme genes in the liver, increased significantly the hepatic expression of MTTP (Ma et al., 2006). Thus, the sustained nuclear localization of ChREBP in the livers from old rats might increase de novo lipogenesis, lipid deposition and VLDL-TAG secretion as demonstrated by the hepatic steatosis and marked hypertryglyceridemia in old rats.

Our study uncovered a novel finding by demonstrating for the first time the permanent nuclear localization of ChREBP independent of the nutritional state in old rats. It has been reported that xylulose 5phosphate, glucose 6-phosphate and fructose 2,6-biphosphate modulates gene expression and translocation of ChREBP to the nucleus (Kabashima et al., 2003; Arden et al., 2012; Dentin et al., 2012; Díaz-Moralli et al., 2012). Besides, a recent report demonstrated that ketone bodies, increasing the binding of 14-3-3 proteins to ChREBP and preventing importin- $\alpha /$ ChREBP interaction, impaired the nuclear import of ChREBP in primary rat hepatocytes (Nakagawa et al., 2013). Although we have not addressed the role of these proteins in the subcellular localization of ChREBP with aging, our data demonstrate that serum ketone bodies levels per se are not able to inhibit the nuclear localization of ChREBP in the liver of overnight and/or prolonged fasted old rats. Thus, it is plausible that importin- $\alpha$ and/or 14-3-3 proteins could be altered in old rats impairing the subcellular translocation of 
ChREBP in the liver. While this suggestion needs further investigation, our data strongly suggest that the adaptation of ChREBP in response to changes in the nutritional state and ketone body levels is impaired with aging (Fig. 7).

In conclusion, the results presented here demonstrate that in fasted, low insulin, old rats, Foxo1 and Foxa2 are localized in the cytoplasm, with a parallel decrease in CPT-1a gene expression and ketone body levels, probably reflecting a reduced mitochondrial long chain fatty acid oxidation and ketogenesis with aging. Besides, we demonstrate a sustained nuclear localization of ChREBP in livers from old rats, even after prolonged fasting, and a significant up-regulation of their lipogenic target genes ACC- $\alpha$ and FAS, favoring hepatic lipid deposition not associated, at least at this stage, with the development of hepatic insulin resistance. Although it is a matter of controversy whether age-dependent liver steatosis might be secondary to increased adiposity and/or insulin resistance, or to aging per se, or to all of these risk factors, our data suggest that the adaptation of key transcription factors involved in lipid metabolism, in response to changes in the nutritional state, is impaired in old rats, and this might contribute to the development of hepatic steatosis with aging.

Supplementary data to this article can be found online at http://dx. doi.org/10.1016/j.exger.2015.05.009.

\section{Acknowledgments}

This work was supported in part by Research Grants PCI08-0136 from Junta de Comunidades de Castilla-La Mancha (JCCM), BFU201239705-C03-01 from Ministerio de Ciencia e Innovación, and S2010/ BMC2423 from Comunidad de Madrid, Spain. A.S. was supported by FPU predoctoral fellowship from Ministerio de Educación y Ciencia (MEC), Spain, and B.B. was supported by CONACyT predoctoral fellowship from México. The Centre of Molecular Biology "Severo Ochoa" is the recipient of institutional aid from the Ramón Areces Foundation. We thank Dr. Perdomo for advice and helpful suggestions and Sergio Moreno for the excellent technical assistance.

\section{References}

Agius, L., Chowdhury, M.H., Davis, S.N., Alberti, K.G.M.M., 1986. Regulation of ketogenesis, gluconeogenesis, and glycogen synthesis by insulin and proinsulin in rat hepatocytes monolayer cultures. Diabetes 35, 286-1293.

Anderson, N., Borlak, J., 2008. Molecular mechanisms and therapeutic targets in steatosis and steatohepatitis. Pharmacol. Rev. 60, 311-357.

Arden, C., Tudhope, S.J., Petrie, J.L., Al-Oanzi, Z.H., Cullen, K.S., Lange, A.J., Towle, H.C., Agius, L., 2012. Fructose 2,6-biphosphate is essential for glucose-regulated gene transcription glucose-6-phospahatase and other ChREBP target genes in hepatocytes. Biochem. J. 443, 111-123.

Benhamed, F., Denechaud, P., Lemoine, M., Robichon, C., Moldes, M., Bertrand-Michel, J., Ratziu, V., Serfaty, L., Housset, C., Capeau, J., Girard, J., Guillou, H., Postic, C., 2012. The lipogenic transcription factor ChREBP dissociates hepatic steatosis from insulin resistance in mice and humans. J. Clin. Invest. 122, 2176-2194.

Biggs III, W.K., Meisenhelder, J., Hunter, T., Cavenee, W.K., Arden, K.C., 1999. Protein kinase b/AKT-mediated phosphorylation promotes nuclear exclusion of the winged helix transcription factor FKHR1. Proc. Natl. Acad. Sci. U. S. A. 96, 7421-7426.

de Solís, A.J., Fernández-Agulló, T., García-SanFrutos, M., Pérez-Pardo, P., Bogónez, E., Andrés, A., Ros, M., Carrascosa, J.M., 2012. Impairment of skeletal muscle insulin action with aging in Wistar rats: role of leptin and caloric restriction. Mech. Ageing Dev. 133, 306-316.

Dentin, R., Pegorier, J.P., Benhamed, F., Foufelle, F., Ferré, P., Fauveau, V., Magnuson, M.A., Girard, J., Postic, C., 2004. Hepatic glucokinase is required for the synergistic action of ChREBP and SREBP-1c on glycolytic and lipogenic gene expression. J. Biol. Chem. 279, 20314-20326.

Dentin, R., Benhamed, F., Hainault, I., Fauveau, V., Foufelle, F., Dyck, J.R., Girard, J., Postic, C., 2006. Liver-specific inhibition of ChREBP improves hepatic statosis and insulin resistance in ob/ob mice. Diabetes 55, 2159-2170.

Dentin, R., Tomas-Cobos, L., Foufelle, F., Leopold, J., Girard, J., Postic, C., Ferré, P., 2012. Glucose 6-phosphate, rather than xylulose 5-phosphate, is required for the activation of ChREBP in response to glucose in the liver. J. Hepatol. 56, 199-209.

Díaz-Moralli, S., Ramos-Montoya, A., Martín, S., Fernandez-Alvarez, A., Casado, M., Cascante, M., 2012. Target metabolomics revealed complementary roles of hexoseand pentose-phosphate in the regulation of carbohydrate-dependent gene expression. Am. J. Physiol. Endocrinol. Metab. 303, E234-E242.
Diraison, F., Moulin, P., Beylot, M., 2003. Contribution of hepatic de novo lipogenesis and reesterification of plasma non esterified fatty acid to plasma tryglycerides synthesis during nonalcoholic fatty liver disease. Diabetes Metab. 29, 478-485.

Eriksson, S., Eriksson, K.F., Bondesson, L., 1986. Nonalcoholic steatohepatitis in obesity: a reversible condition. Acta Med. Scand. 220, 83-88.

Escrivá, F., Agote, M., Rubio, E., Molero, J.C., Pascual-Leone, A.M., Andrés, A., Satrústegui, J., Carrascosa, J.M., 1997. In vivo insulin dependent glucose uptake of specific tissues is decreased during aging in mature Wistar rats. Endocrinology 138, 49-54.

Escrivá, F., Gavete, L., Fermín, Y., Pérez, C., Gallardo, N., Alvarez, C., Andrés, A., Ros, M. Carrascosa, J.M., 2007. Effect of age and moderate food restriction on insulin sensitivity in Wistar rats. Role of adiposity. J. Endocrinol. 194, 131-141.

Farese Jr., R.V., Zechner, R., Newgard, C.B., Walther, T.C., 2012. The problem of establishing relationships between hepatic steatosis and hepatic insulin resistance. Cell Metab. 15, $570-573$.

Fishman, S., Muzumdar, R.H., Atzmon, G., Ma, X., Yang, X., Einstein, F.H., Barzilai, N., 2007. Resistance to leptin action is the major determinant of hepatic triglyceride accumulation in vivo. FASEB J. 21, 53-60.

Flannery, C., Dufour, S., Rabøl, R., Shulman, G.I., Petersen, K.F., 2012. Skeletal muscle insulin resistance promotes increased hepatic de novo lipogenesis, hyperlipidemia, and hepatic steatosis in the elderly. Diabetes 61, 2711-2717.

Gallardo, N., Bonzón-Kulichenko, E., Fernández-Agulló, T., Moltó, E., Gómez-Alonso, S. Blanco, P., Carrascosa, J.M., Ros, M., Andrés, A., 2007. Tissue-specific effects of central leptin on the expression of genes involved in lipid metabolism in liver and white adipose tissue. Endocrinology 148, 5604-5610.

Gan, L., Chitturi, S., Farrell, G.C., 2011. Mechanisms and implications of age-related changes in the liver: nonalcoholic fatty liver diseases in the elderly. Curr. Gerontol. Geriatr. Res. http://dx.doi.org/10.1155/2011/831536.

Goodpaster, B.H., Theriault, R., Watkins, S.C., Kelley, D.E., 2000. Intramuscular lipid content is increased in obesity and decreased by weight loss. Metabolism 49, 467-472.

Horrillo, D., Gallardo, N., Lauzurica, N., Barrús, M.T., San Frutos, M.G., Andrés, A., Ros, M., Fernández-Agulló, T., 2013. Development of liver fibrosis during aging: effects of caloric restriction. J. Biol. Regul. Homeost. Agents 27, 377-388.

Horton, J.D., Goldstein, J.L., Brown, M.S., 2002. SREBPs: activators of the complete program of cholesterol and fatty acid synthesis in the liver. J. Clin. Invest. 109 1125-1131.

Hurtado del Pozo, C., Vesperinas-García, G., Rubio, M.A., Corripio-Sánchez, R., TorresGarcía, A.J., Obregón, M.J., Calvo, R.M., 2011. ChREBP expression in the liver, adipose tissue, and differentiated preadipocytes in human obesity. Biochim. Biophys. Acta 1811, 1194-1200.

Ide, T., Shimano, H., Yahagi, N., Matsuzaka, T., Nakakuki, M., Yamamoto, T., Nakagawa, Y., Takahashi, A., Suzuki, H., Sone, H., Toyoshima, H., Fukamizu, A., Yamada, N., 2004 SREBPs suppress IRS-2 mediated insulin signaling in the liver. Nat. Cell Biol. 6, 351-357.

Ido-Kitamura, Y., Sasaki, T., Kobayashi, M., Kim, H.J., Lee, Y.S., Kikuchi, O., YokotaHashimoto, H., Iizuka, K., Accili, D., Kitamura, T., 2012. Hepatic Foxo1 integrates glucose utilization and lipid synthesis through regulation of ChREBP 0-glycosylation. PLos One 7 (1-7), e47231.

Iizuka, K., Bruick, R.K., Liang, G., Horton, J.D., Uyeda, K., 2004. Deficiency of carbohydrate response element-binding protein (ChREBP) reduces lipogenesis as well as glycolysis. Proc. Natl. Acad. Sci. U. S. A. 101, 7281-7286.

Iizuka, K., Miller, B., Uyeda, K., 2006. Deficiency of carbohydrate-activated transcription factor ChREBP prevents obesity and improves plasma glucose control in leptin-deficient $(o b / o b)$ mice. Am. J. Physiol. Endocrinol. Metab. 291, E358-E364.

Kabashima, T., Kawaguchi, T., Wadzinski, B.E., Uyeda, K., 2003. Xylulose 5-phosphate mediates glucose-induced lipogenesis by xylulose 5 -phosphate-activated protein phosphatase in rat liver. Proc. Natl. Acad. Sci. U. S. A. 100, 5107-5112.

Kamagate, A., Qu, S., Perdomo, G., Su, D., Kim, D.Y., Slusher, S., Meseck, M., Dong, H.H., 2008. Foxo1 mediates insulin-dependent regulation of hepatic VLDL production in mice. J. Clin. Invest. 118, 2347-2364.

Le Lay, J., Kaestner, K.H., 2010. The Fox genes in the liver: from organogenesis to functional integration. Physiol. Rev. 90, 1-22.

Liu, H.Y., Yehuda-Shnaidman, E., Hong, T., Han, J., Pi, J., Liu, Z., Cao, W., 2009. Prolonged exposure to insulin suppresses mitochondrial production in primary hepatocytes. J. Biol. Chem. 284, 14087-14095.

Livak, K., Schmittgen, T., 2001. Analysis of relative gene expression data using real-time quantitative PCR and the 2(-Delta Delta C(T)). Methods 25, 402-408.

Ma, L., Robinson, L.N., Towle, H.C., 2006. ChREBP-Mlx is the principal mediator of glucoseinduced gene expression in the liver. J. Biol. Chem. 281, 28721-28730.

Marchesini, G., Brizi, M., Morselli-Labate, A.M., Bianchi, G., Bugianesi, E., McCullough, A.J. Forlani, G., Melchionda, N., 1999. Association of nonalcoholic fatty liver disease with insulin resistance. Am. J. Med. 107, 450-455.

Moon, Y.A., Liang, G., Xie, X., Frank-Kamenetsky, M., Fitzgerald, K., Koteliansky, V., Brown, M.S., Goldstein, J.L., Horton, J.D., 2012. The Scap/SREBP pathway is essential for developing diabetic fatty liver and carbohydrate-induced hypertriglyceridemia in animals. Cell Metab. 15, 240-246.

Nakagawa, T., Ge, Q., Pawlosky, R., Wynn, R., Veech, R., Uyeda, K., 2013. Metabolite regulation of nucleo-cytosolic trafficking of carbohydrate response element-binding protein (ChREBP): role of ketone bodies. J. Biol. Chem. 288, 28358-28367.

Otero, Y.F., Stafford, J.M., McGuinness, O.P., 2014. Pathway-selective insulin resistance and metabolic disease: the importance of nutrient flux. J. Biol. Chem. 289, 20462-20469.

Sastre, J., Pallardó, F.V., Plá, R., Pellin, A., Juan, G., O'Connor, J.E., Estrella, J.M., Miquel, E., Viña, J., 1996. Aging of the liver: age-associated mitochondrial damage in intact hepatocytes. Hepatology 24, 1199-1205. 
Serrano, R., Villar, M., Gallardo, N., Carrascosa, J.M., Martinez, C., Andrés, A., 2009 The effect of aging on insulin signaling pathway is tissue dependent. Central role of adipose tissue in the insulin resistance of aging. Mech. Ageing Dev. 130, 189-197.

Shimomura, I., Bashmakov, Y., Horton, J.D., 1999. Increased levels of nuclear SREBP-1c associated with fatty livers in two mouse models of diabetes mellitus. J. Biol. Chem. 274, 30028-30032.

Tamura, S., Shimomura, I., 2005. Contribution of adipose tissue and de novo lipogenesis to nonalcoholic fatty liver disease. J. Clin. Invest. 115, 1139-1142.

Trauner, M., Arrese, M., Wagner, M., 2010. Fatty liver and lipotoxicity. Biochim. Biophys. Acta 1801, 299-310.
Wolfrum, C., Stoffel, M., 2006. Coactivation of Foxa2 through PGC1ß promotes liver fatty acid oxidation and triglyceride/VLDL secretion. Cell Metab. 3, 99-110.

Wolfrum, C., Asilmaz, E., Luca, E., Friedman, J.M., Stoffel, M., 2004. Foxa2 regulates lipid metabolism and ketogenesis in the liver during fasting and in diabetes. Nature 432, 1027-1032.

Zhang, W., Patil, S., Chauhan, B., Guo, S., Powell, D.R., Le, A., Klotsas, J., Matika, R., Xiao, X., Franks, R., Heidenreich, K.A., Sajan, M.P., Farese, R.V., Stolz, D.B., Tso, P., Koo, S., Montminy, M., Unterman, T.G., 2006. Foxo1 regulates multiple metabolic pathways in the liver. Effects on gluconeogenic, glycolytic, and lipogenic gene expression. J. Biol. Chem. 281, 10105-10117. 